“C 2018 IEEE. Personal use of this material is permitted. Permission from IEEE must be obtained for all other uses, in any current or future media, including reprinting/republishing this material for advertising or promotional purposes, creating new collective works, for resale or redistribution to servers or lists, or reuse of any copyrighted component of this work in other works." 


\section{Optimizing Inter-operator Network Slicing over Licensed and Unlicensed Bands}

\begin{abstract}
Network slicing has been considered as a key enabling technology for 5G due to its ability to customize and "slice" a common resource to support diverse services and verticals. This paper introduces a novel inter-operator network slicing framework in which multiple mobile network operators (MNOs) can cooperate and jointly slice their accessible spectrum resources in both licensed and unlicensed bands. For the licensed band slicing, we propose the inter-operator spectrum aggregation method which allows two or more MNOs to cooperate and share their licensed bands to support a common set of service types. Since all MNOs enjoy equal rights to access unlicensed bands, we introduce the concept of right sharing for MNOs to share and trade their rights to access unlicensed bands. We develop a modified back-of-the-envelop method for the MNOs to evaluate their value of rights in unlicensed bands when coexisting with other wireless technologies. We develop a network slicing game based on the overlapping coalition formation game to investigate the possible cooperation between MNOs. We prove that our proposed game always has at least one stable slicing structure. We develop a Distributed Alternating Direction Method of Multipliers with Partially Variable Splitting (D-ADMM-PVS) algorithm to implement our proposed framework in a distributed manner. To evaluate the practical performance of our proposed framework, we develop a $\mathrm{C}++-$ based discrete-event simulator and simulate a possible implementation of our proposed framework over 400 base station locations deployed by two primary cellular operators in a major city in Europe. Numerical results show that our proposed framework can almost double the capacity for all supported services for each operator under certain conditions.
\end{abstract}

Index Terms-Network slicing, spectrum sharing, inter-operator, unlicensed band, game theory, ADMM.

\section{INTRODUCTION}

With the proliferation of mobile technologies and emerging wireless services, mobile network operators (MNOs) are experiencing unprecedented growth in wireless data traffic. To meet the future demand in $5 \mathrm{G}$ networks, MNOs have taken steps to secure more spectrum resources. The concept of inter-operator spectrum sharing, also referred to as the co-primary spectrum sharing [1], allows each MNO to share their licensed bands with each other and therefore has the potential to significantly increase the spectrum that is available for each MNO. Both FCC and 3GPP have recently set forth several initiatives that aim at encouraging spectrum sharing among MNOs. More specifically, 3GPP Release 14 promotes the idea of radio access network (RAN) sharing which allows multiple MNOs to share their network resources including infrastructure, network functions, and spectrum resources to reduce their system roll-out cost/delay [2]. FCC also introduced new co-primary shared access rules for several millimeter wave (mmWave) bands to promote cooperation and spectrum sharing among spectrum licensees including Federal governments and MNOs [3]. To further alleviate the spectrum scarcity for commercial cellular use,
MNOs have been allowed to extend their services to unlicensed bands including the $5 \mathrm{GHz}$ unlicensed-national-information-infrastructure (U-NII) radio band [4] as well as the $57-64 \mathrm{GHz}$ and $64-71 \mathrm{GHz}$ bands recently opened by FCC [3].

In addition to supporting more traffic, 5G networks are expected to serve highly heterogenous services with diverse requirements. Network slicing has been considered as a key enabler for $5 \mathrm{G}$, due to its ability to create logical partitions of a common resource. These partitions, known as the network slices, can be orchestrated and customized according to different service requirements. Network slicing has the potential to significantly improve spectrum efficiency and enable more flexible and novel services that cannot otherwise be supported by the existing network architecture.

Allowing multiple MNOs to jointly slice their shared resources in both licensed and unlicensed bands has the following benefits: 1) The spectrum resource that is available to each type of services is now significantly expanded, resulting in increased capabilities to support ultra-high-speed and low-latency services over large bandwidths, 2) It is known that cellular traffic of different operators exhibits significant temporal and spatial variations [5]. Allowing operators to cooperate and compensate each other according to different service demands and requirements would not only improve the spectrum utilization but would also increase their revenues [6], 3) Recent observation showed that the potential inter-operator interference and channel collisions resulting from the selfish behavior of MNOs could result in significant performance degradation for all MNOs. By allowing some MNOs to relinquish their access rights of an unlicensed band to other MNOs in exchange for some forms of compensation, e.g., monetary exchange or a similar treatment in other bands, can reduce contention, improve the spectrum utilization, and increase service reliability.

One key challenge in inter-operator network slicing that remains relatively unexplored is the problem of efficient resource allocation over both licensed and unlicensed bands. Licensed and unlicensed bands exhibit different characteristics and require different mechanisms for spectrum access. In particular, a licensed band is typically allocated to an MNO for exclusive use. Each MNO has already carefully planned its network infrastructure and adopted various centrally controlled resource scheduling and allocation mechanisms to ensure optimal utilization and reliable service support for its user equipments (UEs). The unlicensed band, on the other hand, is open to all wireless technologies. To reduce contention between coexisting systems, current Wi-Fi standards as well as the recently published licensed-assisted access (LAA) protocol rely on a carrier-sense multiple 
access (CSMA)-based channel access mechanism called listen-before-talk (LBT). In this mechanism, both LAA and Wi-Fi transmitters must first sense the channel and can only access it when it is sensed idle. The uncertainty for the channel access in unlicensed bands makes it difficult to support services that require stringent quality-of-service (QoS) guarantees. Therefore, most existing works on network slicing focused on licensed bands. In addition, unlike the licensed band in which MNOs can coordinate and share their exclusively licensed spectrum resources, all MNOs have equal rights to access unlicensed bands. How to share and jointly slice the unlicensed band resources among MNOs is still an open problem.

In this paper, we address the above challenges by designing a novel framework that allows multiple MNOs to jointly divide and share licensed and unlicensed spectrum resources according to the service demands and requirements of their UEs. For licensed band slicing, we propose an inter-operator spectrum aggregation method to allow multiple MNOs to share their licensed band resources. In this method, each MNO divides its licensed band into partitions each of which will be distributed to support a specific type of service. Multiple MNOs can cooperate with each other by aggregating their distributed licensed bands to support the same type of service. We introduce the concept of right sharing to investigate the inter-operator cooperation in unlicensed bands. In this concept, each MNO will first quantify the benefit that can be obtained in unlicensed bands, referred to as the value of rights. MNOs can then negotiate and trade their rights to access unlicensed bands according to the estimated value. We propose a modified back-of-the-envelop ( $\mathrm{mBoE}$ ) method for each MNO to estimate its value of rights as well as the potential value improvement that can be achieved when one or more other MNOs are willing to give up their rights to access unlicensed bands. We observe that if each MNO has been given the choice to slice both licensed or unlicensed bands, the interaction between MNOs can be very complex. For example, if an MNO cannot secure enough resource in the licensed band, it will become more aggressive in unlicensed bands and would like to pay more for the rights of other MNOs. Similarly, if the licensed band can offer sufficient resources to support the required traffic of some MNOs, these MNOs will be more willing to sell their right in unlicensed bands. To investigate the interaction among MNOs, we develop a network slicing game based on overlapping coalition formation game. In this game, MNOs can jointly decide the resource allocation as well as distribution of the utility obtained in each network slice. A network slicing structure can only result in a stable state when no MNO can benefit from unilaterally deviating from this structure. It is known that analyzing an overlapping coalition formation game is notoriously difficult. In particular, such a game does not always have a stable structure. Furthermore, allowing overlaps between coalitions results in infinitely many possible structures which makes exhaustive search-based methods, that are widely used in traditional partition-based coalition formation game,

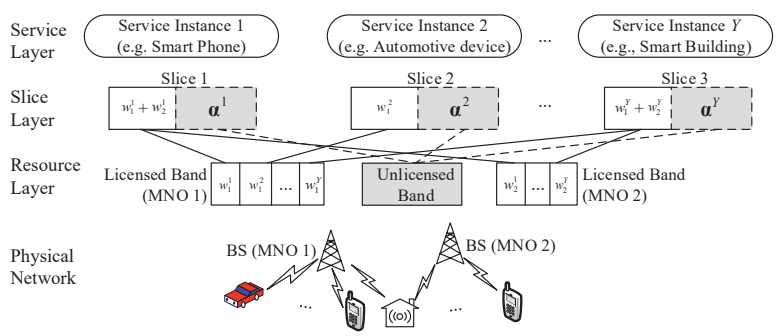

Fig. 1. Inter-operator network slicing over licensed and unlicensed bands. impossible to apply. We prove that our proposed network slicing game always has at least one stable structure. In addition, we develop a novel Distributed Alternating Direction Method of Multipliers with Partial Variable Splitting (D-ADMM-PVS) algorithm to implement our proposed network slicing in a distributed manner. We prove that our proposed algorithm can approach the stable and optimal network slicing structure in linear time without requiring the back-and-forth communication among MNOs. To evaluate the practical performance of our proposed framework, we develop a C++-based discrete-event simulation environment using CSIM development toolkit [7]. We simulate a possible implementation of our proposed framework over 400 base stations (BSs) deployed by two primary cellular network operators in a major city in Europe. Our numerical results show that our proposed framework can almost double the capacity for all supported services for each operator even when only two operators can cooperate with each other.

\section{RELATED WORKS}

Most existing works on inter-operator spectrum sharing focus on licensed band sharing between MNOs with similar traffics and volumes of licensed spectrum resources. More specifically, METIS' future spectrum system concept suggests two co-primary shared access scenarios: limited spectrum pooling (LSP) and mutual renting (MR) [8]. In LSP, two or more MNOs contribute part of their licensed spectrum to form a common pool. All contributing MNOs have equal rights to access the pool and should follow a mutually agreed rule to access the pooled resource. MR allows each MNO to temporally license part or all of its spectrum to another operator. Different from LSP that is equally shared among all the operators, each operator in MR can maintain its strict priority in its own licensed band. Inter-operator resource sharing has also been studied from the network slicing perspective. In [9], a resource allocation mechanism called the Fisher market has been used to study the resource allocation across slices. In [10], a signaling-based network slicing broker solution has been proposed to achieve accurate traffic prediction, slice scheduling, as well as admission control.

Compared to the inter-operator resource sharing in licensed bands, the sharing of unlicensed bands is more complicated. In [11], the authors studied the scenarios that the unlicensed band has been divided into several portions among multiple MNOs. A spectrum sharing scheme was proposed to allow spectrum borrowing and lending among MNOs. Motivated by the recent observations that $\mathrm{Wi}-\mathrm{Fi}$ and LTE coexistence in the unlicensed band could result in $70 \%$ or even $100 \%$ throughput degradation 
for the Wi-Fi systems in the worst case, most existing works focus on developing mechanisms to ensure fair coexistence between LTE and Wi-Fi [12].

III. Network Slicing Framework Design

The main objective of this paper is to design an inter-operator network slicing framework which allows two or more MNOs to jointly slice their accessible spectrum resources to support a common set of $Y$ types of services labeled as $\mathcal{Y}=\{1,2, \ldots, Y\}$. Our framework extends from the 3GPP's active network sharing management architecture introduced in 3GPP Release 14 [2]. In this architecture, a master operator (MOP) collects the global information and manage the allocation of the shared radio resource among participating operators (POPs) via the MOP's network manager (MOP-NM). However, this centralized management approach cannot be directly applied to inter-operator network slicing due to the following reasons: 1) in 3GPP's architecture, MOP monitors and controls a fixed amount of resource shared among a fixed set of POPs. However, in practice, different MNOs can have different demands and requirements of different services. Each MNO may like to cooperate with different subsets of MNOs to support different types of services. 2) 3GPP's architecture only allows sharing of licensed band resources among MNOs. Compared to the licensed bands, unlicensed bands are free and contain much wider bandwidth of spectrum for MNOs to access. Unfortunately, unlicensed bands require different spectrum access mechanism and therefore the licensed band slicing method cannot be extended into unlicensed bands. 3) Allowing the MOP to always collect global information from POPs may result in network congestion and intolerably high latency caused by information collection as well as potential disclosure of proprietary information of POPs.

To address the above issues, this paper introduces a distributed framework in which each MNO decides how much spectrum resource to be distributed for each type of service (service instance) as well as whether to share the distributed resource with other MNOs as illustrated in Figure 1. In our framework, MNOs are self-interested and will only cooperate when an agreement has been reached and mutually agreed among all slices. Our proposed framework jointly optimizes the resource slicing among MNOs according to different service demands and requirements taking into account the different resources and channel access mechanisms in licensed and unlicensed bands.

\section{INTER-OPERATOR NETWORK SLICING A. Network Slicing for Licensed Bands}

We consider a wireless network consisting of a set of $M$ MNOs, labeled as $\mathcal{M}=\{1,2, \ldots, M\}$, that offer services through their network infrastructures, e.g., base stations (BSs). Each MNO is allocated a licensed band $B_{i}$ for exclusive use. Each MNO can support a set of $Y$ types of service for each of its UEs $\mathcal{Y}=\{1,2, \ldots, Y\}$. Each type of service requires a specific minimum QoS guarantee. In this paper, we consider systems with saturated traffic such that each UE can always generate saturated traffic for all supported service types. Let $\eta_{i}^{l}$ be the minimum throughput that needs to be guaranteed for type $l$ service at every UE of MNO $i$.
Each MNO $i$ can divide its licensed band into a set of subcarriers each of which can be allocated to support the traffic for a particular type of service. Generally speaking, the bandwidth of each subcarrier is much smaller than that of the entire licensed band. We can therefore assume the licensed band is continuously dividable among different types of service. Each MNO can divide and aggregate the contiguous and uncontiguous parts of the licensed band to support different services within each MNO using the carrier aggregation technique adopted in existing LTE standards.

Instead of allocating its own licensed band, each MNO can also negotiate with other MNOs to form a group for possible sharing of the licensed bands. We refer a group of MNOs that decide to share their licensed bands with each other to support type $l$ service as a service support group (SSG) denoted as $\mathcal{C}^{l}$ for $\mathcal{C}^{l} \subseteq \mathcal{M}$. Generally speaking, MNOs are self-interested. We therefore assume that each member MNO can evaluate its benefit obtained by cooperating with others and will only form an SSG with others when every member MNO can improve its benefit and also be allocated with a fair sharing of the total utility. The MNOs in an SSG will jointly decide the portion of spectrum resource allocated to the supported service. The spectrum sharing among multiple member MNOs within an SSG can be coordinated by adopting the management architecture for the multiple operator core network (MOCN) specified by 3GPP [2], [13]. In particular, MNOs can jointly decide the spectrum division through the dynamic spectrum management (DSM) block and consult the external spectrum databases for resource distribution.

We introduce the inter-operator spectrum aggregation for MNOs associated with the same SSG to share their licensed band resources for each commonly supported service type $l$. Let $w_{i}^{l}$ be the portion of licensed band distributed by MNO $i$ to support the $l$ th type of service. We have $0 \leq w_{i}^{l} \leq B_{i}$. MNOs in $\mathcal{C}^{l}$ will aggregate their allocated licensed bands for type $l$ service traffic. We can write the total aggregated licensed spectrum allocated by MNOs to support type $l$ service as $w^{l}=\sum_{i \in \mathcal{C}^{l}} w_{i}^{l}$. Each UE associated with a member MNO within an SSG will follow a mutually agreed scheduling procedure to access the aggregated spectrum. The final portion of aggregated spectrum that can be accessed by each UE will depend on the specific network topology as well as traffic from other nearby UEs. Let $\mathcal{L}_{i}$ be the set of all the communication links associated with UEs of MNO $i$. We can write $d_{k, i}^{l}$ as the portion of $w^{l}$ that can be accessed by the $k$ th communication link (e.g., uplink or downlink from each UE or BS) to send data traffic corresponding to type $l$ service, i.e., the total spectrum that can be accessed by each link $k$ of MNO $i$ is given by $d_{k, i}^{l} w^{l}$. We can write the utility obtained by MNO $i$ for serving type $l$ service at the $k$ th link as $\pi_{k, i}^{l}=\rho_{i}^{l} d_{k, i}^{l} w^{l} R_{k, i}$ where $\rho_{i}^{l}$ is the price per data bit charged by MNO $i$ by serving type $l$ service and $R_{k, i}=\log _{2}\left(1+\mathrm{SNR}_{k, i}\right)$ is the throughput per unit $(\mathrm{Hz})$ achieved by link $k$ of MNO $i$ to support type $l$ service and $\mathrm{SNR}_{k, i}$ is the received signal-to-noise ratio for link $k$ when it is the only link to access the channel.

If MNOs can only perform network slicing by jointly 
sharing their licensed bands, we can write the optimization problem for each MNO $i$ as follows:

$$
\begin{aligned}
\max _{\boldsymbol{w}_{i}} & \sum_{k \in \mathcal{L}_{i}} \sum_{l \in \mathcal{Y}} \pi_{k, i}^{l} \\
\text { s.t. } & \sum_{l \in \mathcal{Y}} w_{i}^{l} \leq B_{i} \text { and } d_{k, i}^{l} R_{k, i} \sum_{i \in \mathcal{C}^{l}} w_{i}^{l} \geq \eta_{i}^{l},
\end{aligned}
$$

where $\boldsymbol{w}_{i}=\left\langle w_{i}^{l}\right\rangle_{l \in \mathcal{Y}}$ is the distribution of the licensed band resources decided by MNO $i$.

Our proposed framework can be directly implemented in the co-primary spectrum shared access in METIS' future spectrum system concept with LSP mode [1]. In particular, if MNOs in an SSG decide to operate in the LSP, all the member MNOs will negotiate for a group license and use the inter-operator carrier aggregation strategy to form a common resource pool $w^{l}$ to support type $l$ service.

\section{B. Network Slicing for Unlicensed Band}

In this subsection, we introduce the right sharing framework between MNOs in unlicensed bands.

1) LAA Protocol: Before we discuss the inter-operator spectrum sharing in unlicensed bands, let us first briefly review the CSMA-based LAA protocol. Since the unlicensed band is open to all wireless technologies, to avoid the collision and cross-interference, data transmission is required to follow a LBT-based channel access mechanism. In this mechanism, each UE or BS must first sense the vacancy of the channel for a duration of time called distributed inter-frame spacing (DIFS) plus a random number, referred to as the backoff counter number, of time slots. The value of the backoff counter is uniformly randomly generated between 0 and an integer value called contention window $C W$. The backoff counter is decremented one-by-one for each time slot till zero when the channel is idle. In case that the channel is occupied by other neighboring UE or BS. The backoff counter will be frozen until the channel is sensed to be idle again. Data packets can only be sent if the channel is idle during both DIFS and backoff time.

As observed from the above description, it is generally impossible to guarantee the availability of resources in the unlicensed band, e.g., even the probability of channel access is high, there is still a small chance that an LTE UE or BS cannot send any data packet on the unlicensed band. Let $\xi_{k, i}$ be the probability of channel access for the $k$ th link associated MNO $i$. Let $B^{u}$ be the total amount of spectrum resource of the unlicensed band.

2) Estimation of Probability of Access in Unlicensed Band: Before negotiating with other MNOs, an MNO needs to first pre-evaluate the potential benefit that can be obtained in the unlicensed band. It also needs to identify whether to negotiate with one or more other MNOs for the possibility of giving up their rights in the unlicensed band. Similarly, once an MNO receives a request from another $\mathrm{MNO}$ about giving up its right to access the unlicensed band, it needs to know how much damage it will cause and how much compensation it should expect from the requesting MNOs. In this paper, we assume the benefit for each MNO in unlicensed bands is closely related to its probability of the channel access for each of its UEs.
TABLE I

WI-FI AND LAA CHANNEL ACCESS PARAMETERS [15]

\begin{tabular}{lllll}
\hline & DIFS & $C W_{\min }$ & $C W_{\max }$ & TXOP \\
\hline $802.11 \mathrm{ac}$ & $34 \mathrm{~ms}$ & 3 & 7 & $1.504 \mathrm{~ms}$ \\
\hline LAA & $25 \mathrm{~ms}$ & 3 & 7 & $2 \mathrm{msec}$ \\
\hline
\end{tabular}

We introduce an $\mathrm{mBoE}$ method for each $\mathrm{MNO}$ to pre-evaluate the probability of access for each of its links. The basic idea is to generate a graphical model that can characterize the possible contention among all the intra- and inter-operator channel contentions as well as the channel contentions from other coexisting wireless technologies such as Wi-Fi. Our $\mathrm{mBoE}$ method is extended from the original back-of-the-envelop (BoE) method introduced in [14]. BoE is a simple and effective method that can quickly calculate the probability of access of a contention graph without requiring any detailed information about locations and transmission parameters.

Unfortunately, the original BoE cannot be directly applied into LAA system due to the following reasons: 1) the original BoE method was built on a homogeneous 802.11 network in which all the devices have the same contention parameters. In our system, the LAA BSs and UEs coexist with other wireless technologies such as $\mathrm{Wi}-\mathrm{Fi}, 2$ ) the BoE method needs to have a complete contention graph consisting of all the communication links and the calculation of each link requires to consult the entire network topology. However, in our muti-MNO system, each MNO cannot know the relative locations of UEs or BSs associated with other MNOs. To address these two issues, our $\mathrm{mBoE}$ is built on an empirical table consisting of the premeasured probability of access of each LAA BS or UE when contending with different subsets of Wi-Fi and/or other LAA devices under different network topologies. Compared to the original BoE method, our $\mathrm{mBoE}$ method provides an improved estimation results with reduced computation complexity. In addition, our $\mathrm{mBoE}$ can calculate the probability of access for each local link using only the local network topology.

Before we introduce the detailed method, let us introduce the following assumptions. Note that these assumptions are only used for justifying the $\mathrm{mBoE}$ method and are not necessary for our network slicing game or distribution algorithms introduced later in the paper.

A1) Each UE or BS of one MNO can sense the coexistence of the neighboring UEs and BSs from other MNOs as well as Wi-Fi devices,

A2) The time duration for random backoff countdown is negligible, compared to the duration spent on data transmission,

A3) The distributions for the long-term residual backoff countdown time and transmission time are stationary.

Assumption A1) is reasonable because LAA protocol has different contention/transmission parameters compared to other wireless technologies operated in unlicensed band such as the Wi-Fi (See Table I for a list of transmission parameters of LAA release 13 [15] and 802.11ac Wi-Fi standard). Each UE and BS can monitor the transmission duration of the data packets from other neighboring devices and differentiate Wi-Fi devices, LAA UEs and BSs from other MNOs. Note that the channel fading and shadowing effects may result in the existence of the so called "hidden 
nodes", i.e., some BSs or UEs cannot always successfully detect their neighboring devices. Since our $\mathrm{mBoE}$ method is built on an empirical probability of access table obtained from previous measurements, the effect of the hidden nodes has already been reflected in the measuring results. The impact of the hidden nodes can be further reduced by allowing MNOs to share their sensing results with each other. Each MNO can also extract the local information about channel contenting Wi-Fi devices from the beacon signal broadcasted by Wi-Fi APs to further improve its sensing accuracy. Assumption A2) follows the same observation in [14]. In particular, the countdown time of different links may occur concurrently which in some sense cancels the time spent on resolving the possible collisions among channel contending links. We implement the most recent LAA specification in [15] into our CSIM-based simulator and our experimental results also verify this observation. In other words, the backoff mechanism introduced in the CSMA protocols can successfully avoid the collision among contending devices for most of the time and therefore in most of our measuring results, the data transmission time dominates the channel access time. Assumption 3) has been proved in [16].

The first step of $\mathrm{mBoE}$ method is to establish a contention graph that can capture all the contention between the coexisting devices for each MNO. We formally define contention graph as follows.

Definition 1: A contention graph for a multi-MNO cellular system coexisted in the unlicensed band is a graph $\mathcal{G}=\langle\mathcal{V}, \mathcal{E}\rangle$ comprising a set $\mathcal{V}$ of vertices corresponding to the set of all the coexisting links connecting UEs and BSs associated with all the MNOs as well as the coexisting Wi-Fi links and a set $\mathcal{E}$ of edges each of which connects two vertices that can sense the existence of each other. We also define the contention subgraph associated with MNO $i$ as the subgraph $\mathcal{G}_{i}$ of $\mathcal{G}$ comprising subsets of vertices and edges corresponding to communication links associated with MNO $i$ as well as their sensed entities from other MNOs and Wi-Fi systems.

In Figure 2, we have listed the measured average probability of access under different contention topologies using our developed CSIM-based simulator. Note that since the LAA Release 13 only supports downlink transmission in unlicensed bands, the number of possible contention graphical topologies that involves each BS should be limited, e.g., if all the LAA transmitters correspond to BSs deployed by MNOs, the maximum number of BSs contending with each other in each local area will be equivalent to the number of MNOs. In addition, as observed in many existing results that as the number of channel contending devices becomes large, the probability of channel access will drop significantly. Therefore, it is unnecessary for each UE or BS to maintain a table that includes a large number of coexisting devices.

Note that in Figure 2 we observe a significant drop on the probability of access for Wi-Fi APs in our results presented. This is because we have adopted the most recent LAA specification in Table I in which the LAA BS has a shorter DIFS waiting time as well as longer TXOP transmission

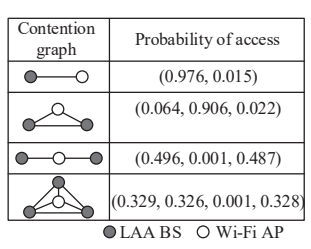

Fig. 2. Table of contention subgraphs and the corresponding probability of access measured by our CSIM simulator.

duration compared to the parameters specified in 802.11ac standard. Our observation is similar to that reported in [16].

We also define the maximum independent set for an MNO $i$ as follows:

Definition 2: An independent set associated with MNO $i$ is a set of vertices in $\mathcal{G}_{i}$ in which no two of which are adjacent. A maximum independent set for MNO $i$ is an independent set of largest possible size for graph $\mathcal{G}_{i}$.

The maximum independent sets can be found by standard approaches in polynomial time [17].

One of the main idea behind the above procedure is that the maximum independent sets dominate the possible channel contention as well as channel access among all the entities from different MNOs in the same coverage area. In particular, the following proposition has been proved in [14].

Proposition 1: [14, Propositions 1] A CSMA-based system spends most of its time in the maximum independent sets and very little time in other states.

We can write the vector for the probability of access for all links associated with MNO $i$ as $\boldsymbol{\xi}_{i}=\left\langle\xi_{k, i}\right\rangle_{k \in \mathcal{L}_{i}}$.

Each MNO can then use the following procedure to estimate the probability of access for each of its links:

P1) Establish a contention subgraph $\mathcal{G}_{i}$ in the unlicensed band using the sensing results from all the corresponding UEs and BSs of MNO $i$,

P2) Each MNO $i$ can then identify the possible maximum independent sets for $\mathcal{G}_{i}$ using standard approaches,

P3) Each MNO $i$ generates a modified subgraph $\mathcal{G}_{i}^{\prime}$ by removing all the vertices that are not associated with any maximum independent set from $\mathcal{G}_{i}$,

P4) Each MNO $i$ searches for the probability of channel access $\xi_{k, i}$ for each link $k$ from the pre-stored contention subgraph table.

Since each MNO can detect the contention from other MNOs, it can also estimate the possible improvement of the channel access probability if one or more other MNOs stop accessing the unlicensed band. We define the estimated contention subgraph $\mathcal{G}_{i \backslash j}$ for MNO $i$ when MNO $j$ stops accessing the unlicensed band as the subgraph of $\mathcal{G}_{i}$ such that all vertices associated with links from MNO $j$ are removed for $i \neq j$. By replacing graph $\mathcal{G}_{i}$ with subgraph $\mathcal{G}_{i \backslash j}$ in procedure P1), MNO $i$ can estimate the resulting probability of channel access $\xi_{k, i \backslash j}$ for each of its links following procedures $\mathrm{P} 2$ ) to $\mathrm{P} 4$ ). We write the vector of channel access probabilities for all the links associated with MNO $i$ when MNO $j$ stops accessing the unlicensed band as $\boldsymbol{\xi}_{i \backslash j}=\left\langle\xi_{k, i \backslash j}\right\rangle_{k \in \mathcal{L}_{i}}$ for $i \neq j$.

We have verified the estimated probability of access calculated from the procedures P1)-P4) using our developed 
CSIM simulator under different network topologies in [18]. Please see our technical report in [18] for more detailed justification of our proposed $\mathrm{mBoE}$ method.

3) Inter-operator Right Sharing: As mentioned previously, the performance degradation of an MNOs for giving up its right to access the unlicensed band should be compensated by all the other MNOs that are benefit from the reduction of channel contention. A mutual agreement must be reached by the right-giving-up MNOs and the MNOs that are willing to provide compensations. Let $\mathcal{D}$ be the set of MNOs that are willing to give up their rights to access the unlicensed band for $\mathcal{D} \subset \mathcal{M}$. How to divide the utility between the right-givingup MNOs and the rest of the MNOs depends on the detailed requirements and utility improvement that can be achieved by each MNO. In this paper, we employ a transferrable utility framework in which the utility obtained by the MNOs in the unlicensed band can be freely transferred between different member MNOs. We will give a more detailed description about this framework in the next section.

From the previous discussion, the unlicensed band resources that can be accessed by the $k$ th link of MNO $i$ is specified by the probability of channel access $\xi_{k, i \backslash \mathcal{D}}$. Each MNO can then distribute the channel access at each link according to the QoS of the supported types of services. Let $\alpha_{k, i}^{l}$ be the portion of the channel access probability that is allocated to support type $l$ service at link $k$ of MNO $i$. We have $\sum_{l \in \mathcal{Y}} \alpha_{k, i}^{l}=$ $\xi_{k, i \backslash \mathcal{D}}$. We also write $\boldsymbol{\alpha}_{i}=\left\langle\alpha_{k, i}^{l}\right\rangle_{k \in \mathcal{L}_{i}, l \in \mathcal{Y}}$. We can write the utility obtained by MNO $i$ from supporting type $l$ service at the $k$ th link as $\nu_{k, i}^{l}=\rho_{i}^{l} \alpha_{k, i}^{l} B^{u} R_{k, i}$. We can write the resource allocation problem in unlicensed bands as

$$
\begin{aligned}
& \max _{\boldsymbol{\alpha}_{i}} \sum_{k \in \mathcal{L}_{i}} \sum_{l \in \mathcal{Y}} \nu_{k, i}^{l} \\
& \text { s.t. } \sum_{l \in \mathcal{Y}} \alpha_{k, i}^{l}=\xi_{k, i \backslash \mathcal{D}} \text { and } \alpha_{k, i}^{l} B^{u} R_{k, i} \geq \eta_{i}^{l}, \quad \forall k \in \mathcal{L}_{i} .
\end{aligned}
$$

\section{Network Slicing over Licensed and Unlicensed Bands}

It can be observed that the network slicing decision made by each MNO in the licensed and unlicensed bands can be closely related to each other. In particular, if an MNO cannot secure enough spectrum resource in the licensed band, it will become more aggressive in the unlicensed band and would like to pay more for the right of other MNOs. Similarly, if the licensed band can offer sufficient resources to support the required traffic of some MNOs, these MNOs will be more willing to sell their right in the unlicensed band.

The main objective for each MNO is to carefully decide the resource distributed in both licensed and unlicensed bands for each slice. Let $\varpi_{k, i}^{l}=\pi_{k, i}^{l}+\nu_{k, i}^{l}$. Each MNO $i$ decides the optimal resource distribution by solving the following problem:

$$
\begin{aligned}
\max _{\boldsymbol{w}_{i}, \boldsymbol{\alpha}_{i}} & \sum_{k \in \mathcal{L}_{i}} \sum_{l \in \mathcal{Y}} \varpi_{k, i}^{l} \\
\text { s.t. } & \sum_{l \in \mathcal{Y}} \alpha_{k, i}^{l}=\xi_{k, i \backslash \mathcal{D}} \text { and } \sum_{l \in \mathcal{Y}} w_{i}^{l} \leq B_{i}, \\
& \left(d_{k, i}^{l} \sum_{i \in \mathcal{C}^{l}} w_{i}^{l}+\alpha_{k, i}^{l} B^{u}\right) R_{k, i} \geq \eta_{i}^{l} .
\end{aligned}
$$

\section{Network Slicing Game}

To model the network slicing problem among multiple MNOs, we use the framework of the overlapping coalition formation game. The overlapping coalition formation game attracts much attention recently due to its capability to investigate the resource allocation problem between multiple players that can allocate different portions of their resources to simultaneously support different services as members of different coalitions [19]. Compared to the traditional partition-based coalition formation game, allowing players to interaction with each other across multiple coalitions has the potential to further improve the resource utilization efficiency and increase the outcome for the players.

We formally define network slicing game as follows.

Definition 3: A network slicing game is defined by the tuple $\mathcal{A}=\langle\mathcal{M}, \boldsymbol{B}, \mathcal{Y}, \varpi\rangle$ where $\mathcal{M}$ is a set of MNOs that are the players of the game, $\boldsymbol{B}=\cup_{i \in \mathcal{M}} B_{i} \times B^{u}$ is the spectrum resources that can be accessed by each MNO in both licensed and unlicensed band, $\mathcal{Y}$ is the set of service types for each MNO to distribute resources, $\varpi$ is the vector of utilities that can be obtained by the MNOs.

We give a more detailed discussion for each of the above elements in the network slicing game as follows. Each MNO can access resources in both licensed and unlicensed bands. The licensed band that can be accessed by each MNO includes both its own licensed band as well as the licensed bands owned by other MNOs that can be potentially aggregated by the MNO. Each MNO can also access the resources in the unlicensed band through channel contention. The main objective for MNOs is to slice the available resource to support all types of service. Each type $l$ of service is specified by a threshold $\eta_{i}^{l}$ which characterizes the minimum QoS that should be guaranteed by each MNO $i$ and a price $\rho_{i}^{l}$ describing the unit price charged by MNOs for supporting the service. A slice $c^{l}$ is a vector $c^{l}=\left\langle c_{1}^{l}, c_{2}^{l}, \ldots, c_{M}^{l}\right\rangle$ where $c_{i}^{l}$ is the resource allocated by MNO $i$ to support type $l$ service. Each slice comprises of spectrum resources from the licensed and unlicensed band. The licensed band resource distributed to support type $l$ service is given by $\boldsymbol{w}^{l}=\left\langle w_{i}^{l}\right\rangle_{i \in \mathcal{C}^{l}}$. Each MNO can also access the unlicensed band with a certain probability of access. The unlicensed band resource distributed to support type $l$ service can be written as $\boldsymbol{\alpha}^{l}=\left\langle\alpha_{k, i}^{l}\right\rangle_{k \in \mathcal{L}_{i}, i \in \mathcal{M}}$. $\alpha_{k, i}^{l}=0$ means that MNO $i$ does not allocate any unlicensed band resource to support type $l$ service for link $k$. We define a network slicing structure $\boldsymbol{c}=\left\langle\boldsymbol{c}^{l}\right\rangle_{l \in \mathcal{Y}}$ as a vector specifying the resource allocations for all the MNOs among all types of service.

We consider a game with transferrable utility in which the utility obtained in a slice can be freely transferred among member MNOs. A characterization function maps each slice of MNOs into a single value referred to as the worth of a slice. The worth characterizes the total utility that is available to all the contributing MNOs. The worth for each slice consists of utilities obtained from both licensed and unlicensed bands. We can write the worth of a slice $c^{l}$ as $v\left(c^{l}\right)=\sum_{i \in \operatorname{supp}\left(c^{l}\right)} \sum_{k \in \mathcal{L}_{i}} \varpi_{k, i}^{l}$ where supp is the support. 
We can observe that the worth function is monotone, i.e., $v\left(c^{l}\right) \geq v\left(c^{l^{\prime}}\right)$ for any $c^{l}, c^{l^{\prime}}$ such that $c_{i}^{l} \geq c_{i}^{l^{\prime}}$ for all $i \in$ $\mathcal{M}$. In other words, MNOs will always use all the accessible resources to serve the supported service.

We define an allocation of utility for each slice as $x^{l}=$ $\left\langle x_{i}^{l}\right\rangle_{i \in \operatorname{supp}\left(c^{l}\right)}$ which describes the worth distributed among all the MNOs. $\boldsymbol{x}^{l}$ is efficient if $\sum_{i \in \operatorname{supp}\left(\boldsymbol{c}^{l}\right)} x_{i}^{l}=v\left(\boldsymbol{c}^{l}\right) . \boldsymbol{x}^{l}$ is also called imputation if it is efficient and satisfies the individual rationality, i.e., $x_{i}^{l} \geq v\left(\dot{c}_{i}^{l}\right)$ where $\dot{c}_{i}^{l}$ is the slice for type $l$ service if MNO $i$ did not cooperate with another other MNOs in both licensed and unlicensed bands. We refer to a network slicing agreement as a tuple $\langle\boldsymbol{c}, \boldsymbol{x}\rangle$ where $\boldsymbol{x}=\left\langle\boldsymbol{x}^{l}\right\rangle_{l \in \mathcal{Y}}$.

As mentioned earlier, MNOs are self-interest entities and always seek to maximize their individual utilities by forming coalitions with different MNOs in both licensed and unlicensed bands. However, the resource distribution and negotiation among MNOs across different slices can be very complex. For example, when an MNO negotiating with another MNO for sharing their resources to serve a specific type of service, it can also offer a certain term that may affect the cooperation with other MNOs in serving other types of service. Similarly, when an MNO deviates from a network slicing agreement with another MNO in serving a specific type of service, it can also affect its cooperation with other MNOs in other service types. The main solution concept in the network slicing game is the core. We extend the concept of the conservative core in the overlapping coalition formation game into our network slicing game.

Definition 4: Given a network slicing game $\mathcal{A}=\langle\mathcal{M}, \boldsymbol{B}, \mathcal{Y}, \varpi\rangle$ and a subset of MNOs $\mathcal{N} \subseteq \mathcal{M}$. Suppose $\langle\boldsymbol{c}, \boldsymbol{x}\rangle$ and $\left\langle\boldsymbol{c}^{\prime}, \boldsymbol{x}^{\prime}\right\rangle$ are two network slicing agreements such that for any slice $c^{l} \in c$ either $\operatorname{supp}\left(\boldsymbol{c}^{l}\right) \subseteq \mathcal{N}$ or $\operatorname{supp}\left(\boldsymbol{c}^{l}\right) \subseteq \mathcal{M} \backslash \mathcal{N}$. We say that network slicing agreement $\left\langle\boldsymbol{c}^{\prime}, \boldsymbol{x}^{\prime}\right\rangle$ is a profitable deviation of $\mathcal{N}$ from $\langle\boldsymbol{c}, \boldsymbol{x}\rangle$ if for all $j \in \mathcal{N}$, we have $\varpi_{j}\left(\boldsymbol{c}^{\prime}, \boldsymbol{x}^{\prime}\right)>\varpi_{j}(\boldsymbol{c}, \boldsymbol{x})$. We say that a network slicing agreement $\langle\boldsymbol{c}, \boldsymbol{x}\rangle$ is in the core of $\mathcal{A}$ if no subset of $\mathcal{N}$ has a profitable deviation from it. In other words, for any subsets of MNOs $\mathcal{N} \subseteq \mathcal{M}$, any network slicing structure $c_{\mathcal{N}}$, and any imputation $\boldsymbol{x}^{\prime}$, we have $\varpi_{j}\left(\boldsymbol{c}^{\prime}, \boldsymbol{x}^{\prime}\right) \leq \varpi_{j}(\boldsymbol{c}, \boldsymbol{x})$.

We have the following result.

Theorem 1: The core of the network slicing game is non-empty and any outcome in the core maximizes the social welfare.

Proof: See technical report [18] for the proof.

\section{Distributed Optimization}

One of the main challenges for the inter-operator spectrum sharing is to minimize the communication overhead between MNOs. In this section, we propose a simple and distributed algorithm framework that can achieve the stable and optimal network slicing structure that is in the core. Our algorithm is based on the distributed ADMM [20] algorithm to decompose the optimization problem into a set of subproblems. Unfortunately, it is known that the traditional ADMM method can only solve problems consisting of two blocks of random variables and therefore cannot be directly applied to solve problem (3) consisting of a large number of variables. In addition, the original ADMM method is a centralized approach that requires all players to reveal their private information to a central controller. Most existing distributed ADMM methods focused on designing a consensus mechanism in which the neighboring agents can exchange and jointly update a local copy of their model parameters [21]. These methods cannot be directly applied to solve network slicing problem in inter-operator systems because MNOs are generally unwilling to share their private proprietary information with each other.

We propose a D-ADMM-PVS algorithm to optimize the network slicing for inter-operator systems. In our algorithm framework, the inter-operator network slicing problem is first divided into $\sum_{i \in \mathcal{M}}\left|\mathcal{L}_{i}\right|$ number of sub-problems each of which can be solved by an individual link (can be either UE or BS of the corresponding link) of an MNO using its local information. Each link will submit a single dual variable to its associated MNO and all the MNO will only coordinate their collected dual variable using a linear function.

As observed in Section V, the property of transferrable utility makes MNOs have the incentive to jointly slice their resources and maximize the total social welfare. Let us write the social welfare maximization problem for the network slicing game as follows.

$$
\begin{aligned}
\max _{\boldsymbol{w}, \boldsymbol{\alpha}} & \sum_{i \in \mathcal{M}} \sum_{k \in \mathcal{L}_{i}} \sum_{l \in \mathcal{Y}} \varpi_{k, i}^{l} \\
\text { s.t. } & \sum_{l \in \mathcal{Y}} \alpha_{k, i}^{l}=\xi_{k, i \backslash \mathcal{D}}, \sum_{l \in \mathcal{Y}} w_{i}^{l} \leq B_{i}, \\
& \left(d_{k, i}^{l} \sum_{i \in \mathcal{M}} \boldsymbol{w}_{i}+\boldsymbol{\alpha}_{k, i} B^{u}\right) R_{k, i} \succeq \boldsymbol{\eta}_{i}, \\
& 0 \leq \alpha_{k, i}^{l} \leq 1 \text { and } 0 \leq w_{i}^{l} \leq 1, \forall i \in \mathcal{M},
\end{aligned}
$$

where $\boldsymbol{\alpha}=\left\langle\boldsymbol{\alpha}_{k, i}\right\rangle_{k \in \mathcal{L}_{i}, i \in \mathcal{M}}, \quad \boldsymbol{\alpha}_{k, i}=\left\langle\alpha_{k, i}^{l}\right\rangle_{l \in \mathcal{Y}}$, and $\boldsymbol{w}=\left\langle\boldsymbol{w}_{i}\right\rangle_{i \in \mathcal{M}}, \boldsymbol{w}_{i}=\left\langle w_{i}^{l}\right\rangle_{l \in \mathcal{Y}}, \boldsymbol{\eta}_{i}=\left\langle\eta_{i}^{l}\right\rangle_{l \in \mathcal{Y}}$ is vector of the minimum QoS required by type $l$ service supported by MNO $i$, and $\succeq$ is the vector inequality.

Note that in problem (4), we replace $\operatorname{supp}\left(\boldsymbol{c}^{l}\right)$ with the set of all the MNOs $\mathcal{M}$. This does not impact our results because the utility division among MNOs in each slice has already been decided by $d_{k, i}^{l}$. In other words, even if, due to the limit of the resources, some MNOs choose to not distribute any licensed resource to support a certain type of services (e.g., type $l$ service), this does not mean these MNOs cannot receive benefit from serving type $l$ services for its UEs because they can still access the spectrum resource $\boldsymbol{w}^{l}$ distributed by other MNOs. In other words, $w_{i}^{l}=0$ does not mean $\pi_{k, i}^{l}=0$.

Let $f_{k, i}\left(\boldsymbol{\alpha}_{k, i}\right)=\sum_{l \in \mathcal{Y}} B^{u} \rho_{i}^{l} R_{k, i} \alpha_{k, i}^{l}, \quad$ and $g(\boldsymbol{w})=\sum_{i \in \mathcal{M}} \sum_{k \in \mathcal{L}_{i}}\left(\sum_{l \in \mathcal{Y}} \rho_{i}^{l} R_{k, i} d_{k, i}^{l}\left(\sum_{i \in \mathcal{M}} w_{i}^{l}\right)\right)$. We can rewrite the objective function in (4a) as the summation of a set of sub-functions as follows

$$
\sum_{i \in \mathcal{M}} \sum_{k \in \mathcal{L}_{i}} f_{k, i}\left(\boldsymbol{\alpha}_{k, i}\right)+g(\boldsymbol{w}) .
$$

Let us introduce a set of indicator functions to incorporate constraints (4b) into the objective function. For the separable 
constraints (4b), we have

$\mathcal{I}_{k, i}^{\alpha}\left(\boldsymbol{\alpha}_{k, i}\right)=\left\{\begin{array}{ll}0, & \boldsymbol{\alpha}_{k, i} \in \mathcal{E}_{k, i}^{\alpha}, \\ \infty, & \boldsymbol{\alpha}_{k, i} \notin \mathcal{E}_{k, i}^{\alpha},\end{array}, \mathcal{I}^{w}(\boldsymbol{w})= \begin{cases}0, & \boldsymbol{w} \in \mathcal{E}^{w}, \\ \infty, & \boldsymbol{w} \notin \mathcal{E}^{w},\end{cases}\right.$

where

$$
\begin{aligned}
\mathcal{E}_{k, i}^{\alpha} & =\left\{\boldsymbol{\alpha}_{k, i} \mid \sum_{l \in \mathcal{Y}} \alpha_{k, i}^{l}=\xi_{k, i \backslash \mathcal{D}}, 0 \leq \alpha_{k, i}^{l} \leq 1\right\}, \\
\mathcal{E}^{w} & =\left\{\boldsymbol{w} \mid \sum_{l \in \mathcal{Y}} w_{i}^{l} \leq B_{i}, 0 \leq w_{i}^{l} \leq 1, \forall i \in \mathcal{M}\right\},
\end{aligned}
$$

and we can incorporate them into objective functions as follows:

$f_{k, i}^{+}\left(\boldsymbol{\alpha}_{k, i}\right)=f_{k, i}\left(\boldsymbol{\alpha}_{k, i}\right)+\mathcal{I}_{k, i}^{\alpha}, g^{+}(\boldsymbol{w})=g(\boldsymbol{w})+\mathcal{I}^{w}(\boldsymbol{w})$.

For the inseparable constraint (4c), we also introduce an indicator function as follows

$$
\mathcal{I}_{Z}(\boldsymbol{X})= \begin{cases}0, & \boldsymbol{X} \in \mathcal{E}_{Z}, \\ \infty, & \boldsymbol{X} \notin \mathcal{E}_{Z},\end{cases}
$$

where $\boldsymbol{X}=(\boldsymbol{\alpha}, \boldsymbol{w})$, and

$$
\mathcal{E}_{Z}=\left\{\boldsymbol{X} \mid\left(d_{k, i}^{l} \sum_{i \in \mathcal{M}} \boldsymbol{w}_{i}+\boldsymbol{\alpha}_{k, i} B^{u}\right) R_{k, i} \succeq \boldsymbol{\eta}_{i}\right\} .
$$

We can then reformulate the above optimization problem in (4) as

$$
\begin{array}{rl}
\max _{\boldsymbol{X}, \boldsymbol{Z}} & F(\boldsymbol{X})+\mathcal{I}_{\boldsymbol{Z}}(\boldsymbol{Z}), \\
\text { s.t. } & \boldsymbol{X}-\boldsymbol{Z}=\mathbf{0},
\end{array}
$$

where $F(\boldsymbol{X})=\sum_{i \in \mathcal{M}} \sum_{k \in \mathcal{L}_{i}} f_{k, i}^{+}\left(\boldsymbol{\alpha}_{k, i}\right)+g^{+}(\boldsymbol{w})$, and $\boldsymbol{Z}$ is the auxiliary variable introduced to isolate the inseparable constraint. The augmented Lagrangian of problem (12) is

$\mathcal{L}_{\gamma}(\boldsymbol{X}, \boldsymbol{Z}, \boldsymbol{\Lambda})=F(\boldsymbol{X})+\mathcal{I}_{Z}(\boldsymbol{Z})+\boldsymbol{\Lambda}^{T}(\boldsymbol{X}-\boldsymbol{Z})+\frac{\gamma}{2}\|\boldsymbol{X}-\boldsymbol{Z}\|_{2}^{2}$,

where $\vartheta>0$ is the augmented Lagrangian parameter, and $\boldsymbol{\Lambda}$ is the dual variable. We can then follow the same line as standard ADMM and write the centralized solution for (12) as follows:

$$
\begin{aligned}
\boldsymbol{X}^{(t+1)} & :=\arg \min _{\boldsymbol{X}} F(\boldsymbol{X})+\frac{\vartheta}{2}\left\|\boldsymbol{X}-\boldsymbol{Z}^{(t)}+\boldsymbol{\Lambda}^{(t)}\right\|_{2}^{2} \\
\boldsymbol{Z}^{(t+1)} & :=\arg \min _{\boldsymbol{Z}} \mathcal{I}_{Z}(\boldsymbol{Z})+\frac{\vartheta}{2}\left\|\boldsymbol{X}^{(t+1)}-\boldsymbol{Z}+\boldsymbol{\Lambda}^{(t)}\right\|_{2}^{2} \\
\boldsymbol{\Lambda}^{(t+1)} & =\boldsymbol{\Lambda}^{(t)}+\boldsymbol{X}^{(t+1)}-\boldsymbol{Z}^{(t+1)}
\end{aligned}
$$

where we use superscript $(t)$ to denote the $t$ th iteration.

To solve the above problem in a distributed manner, we split variable $\boldsymbol{X}$ into a set of sub-vectors, namely $\boldsymbol{\alpha}_{k, i}$ and $\boldsymbol{w}_{i}$. We also separate the $\boldsymbol{X}$-updating step into a set of sub-problems as follows.

Each link $k$ solves the following $\boldsymbol{\alpha}_{k, i}$-subproblem for unlicensed band resource distribution using its local information:

$$
\boldsymbol{\alpha}_{k, i}^{(t+1)}=\arg \min _{\boldsymbol{\alpha}_{k, i}} f_{k, i}^{+}\left(\boldsymbol{\alpha}_{k, i}\right)+\frac{\vartheta}{2}\left\|\boldsymbol{\alpha}_{k, i}-\boldsymbol{Z}_{k, i}^{(t)}+\boldsymbol{\Lambda}_{k, i}^{(t)}\right\|_{2}^{2} ;
$$

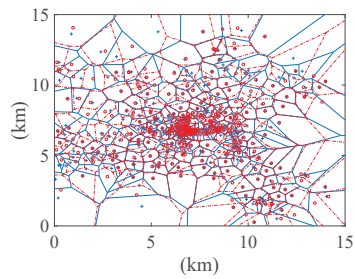

Fig. 3. Distribution of BSs for two cellular MNOs.

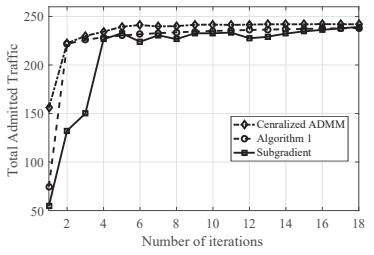

Fig. 4. Convergence performance of Algorithm 1.

MNOs will jointly solve the following $\boldsymbol{w}$-subproblem for licensed band resource distribution through DSM function block in the 3GPP network sharing framework:

$$
\boldsymbol{w}^{(t+1)}=\arg \min _{\boldsymbol{w}} g^{+}(\boldsymbol{w})+\frac{\vartheta}{2}\left\|\boldsymbol{w}-\boldsymbol{Z}_{w}^{(t)}+\boldsymbol{\Lambda}_{w}^{(t)}\right\|_{2}^{2} ;
$$

A coordinator deployed in the DSM block is responsible for coordinating the $\boldsymbol{Z}$-updating in (14b) and the $\boldsymbol{\Lambda}$-updating in (14c). We summarize the details of the proposed algorithm in Algorithm 1. The convergence rate is presented in Theorem 2.

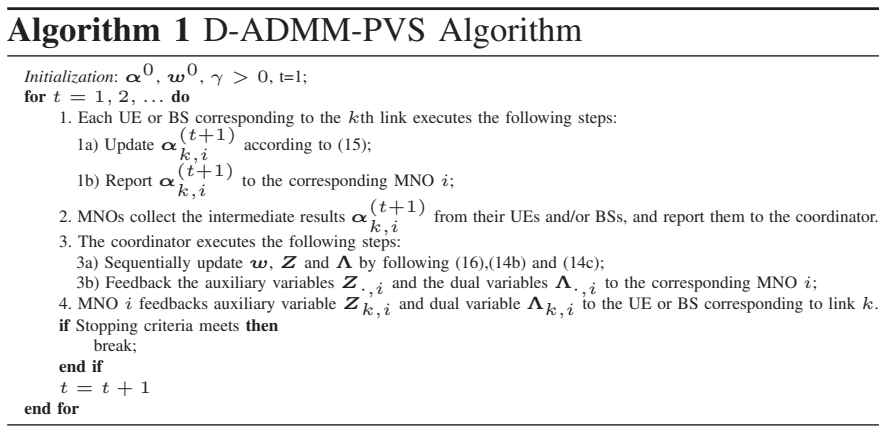

We can prove the following result.

Theorem 2: The augmented Lagrangian form of the objective function for problem (12a) is separable and convex. Algorithm 1 maximizes the social welfare.

Proof: See technical report [18] for the proof.

\section{PERFORMANCE EVALUATION}

We develop a C++-based discrete event simulator using the CSIM development toolkit [7] with total 3000+ lines of codes to simulate the scheduling and contention behavior between MNOs as well as that between the LAA system and Wi-Fi devices over licensed and unlicensed bands. We simulate the possible implementation of our proposed inter-operator network slicing framework over $400 \mathrm{BSs}$ (including GSM and UMTS BSs) deployed by two primary cellular MNOs in one of the major cities in Europe. The locations and coverage areas of BSs deployed by both MNOs are presented in Figure 3. We consider two types of services (e.g., video and audio streaming) requiring $10 \mathrm{Mbps}$ and $20 \mathrm{Mbps}$ minimum guaranteed throughput. We present more simulation results in our technical report [18].

We first compare the convergence performance of Algorithm 1 with other existing approaches for our simulated system in Figure 4. We can observe that Algorithm 1 can converge to the optimal network slicing solution within the first few iterations (less than 14 iterations in both cases). We also present the convergence rate when a centralized ADMM in [20] can be implemented to control the network slicing in a centralized 


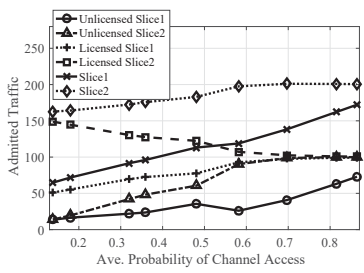

Fig. 5. Traffic admitted by each slice under different network densities.

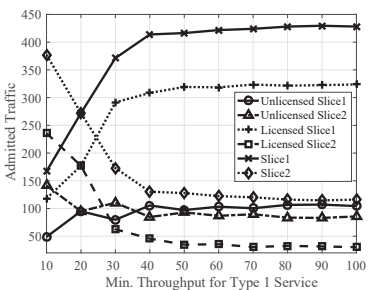

Fig. 7. Traffic admitted by each Fig. 8. Traffic admitted by slice under different min throughput network slicing under different min guarantees.

throughput guarantees.

fashion. In this case, a centralized controller (deployed in a shared radio access network according to 3GPP specification) can collect all the information from all the MNOs and calculate resource distribution for each slice among all the MNOs. It can be easily observed that this implementation will result in a large communication overhead between MNOs. We can observe that our proposed Algorithm 1 presents a very similar convergence performance as the centralized approach and can approach a neighborhood of the optimal solution within first 2 iterations which is much faster than the subgradient method.

We consider 9 regions from the rural areas to the city center with different BS deployment densities to evaluate the impact of the network density on the performance of network slicing. The decrease of the network density also results in the increase of the probability of access for all LAA links. We compare the admitted traffics for different slices under different average channel access probability for the UEs in Figures 5 and 6 . We observe that allowing MNOs to jointly access licensed and unlicensed bands can significantly increase the traffic volume admitted for all the supported services. Interestingly, we can observe that the portion of the admitted traffic for type 1 service decreases with the channel access probability in unlicensed band. This is because the unlicensed band is free and hence when the channel access probability becomes high, it is more economic for MNOs to offload traffic from licensed band to unlicensed band.

In Figures 7 and 8, we fix the minimum throughput that needs to be guaranteed for type 2 service to $\eta_{i}^{2}=20 \mathrm{Mbps}$ and compare traffic volumes admitted by network slicing under different throughput guarantees for type 1 service. We observe that the traffic admitted by type 2 service decreases with the minimum throughput requirement of type 1 service. This is because the MNOs tend to obtain more benefit from the services with a higher requirement. Therefore, when the minimum throughput required by type 1 service increases from $10 \mathrm{Mbps}$ to $100 \mathrm{Mbps}$, both MNOs will distribute more resources to the slice that supports type 1 service.

\section{CONCLUSION}

This paper investigates the inter-operator network slicing over licensed and unlicensed bands. We develop inter-operator spectrum aggregation method for licensed band slicing and a right sharing concept for licensed band slicing between MNOs. The inter-operator network slicing problem has then been formulated as an overlapping coalition formation game. We develop a distributed optimization algorithm based on D-ADMM-PVS to implement inter-operator network slicing. To evaluate the practical performance of our proposed framework, we develop a C++-based discrete-event simulator. We also employed the real distribution of BSs deployed by two cellular MNOs to simulate the possible implementation of inter-operator network slicing in an urban city environment. Our numerical results show that our proposed network slicing framework significantly increases the admitted traffics for all supported services.

\section{REFERENCES}

[1] B. Singh, S. Hailu, K. Koufos, A. A. Dowhuszko, O. Tirkkonen, R. Jntti, and R. Berry, "Coordination protocol for inter-operator spectrum sharing in co-primary $5 \mathrm{~g}$ small cell networks," IEEE Communications Magazine, vol. 53, no. 7, pp. 34-40, July 2015.

[2] 3GPP, "Telecommunication management; network sharing; concepts and requirements," 3GPP TS 32.130, Jun. 2016.

[3] Federal Communications Commission, "Report and Order and Further Notice of Proposed Rule Making," no. 16-89, Jul. 2016.

[4] "LTE in unlicensed spectrum: Harmonious coexistence with Wi-Fi," White Paper, Qualcomm, 2014.

[5] U. Paul, A. P. Subramanian, M. M. Buddhikot, and S. R. Das, "Understanding traffic dynamics in cellular data networks," in IEEE INFOCOM, Shanghai, China, April 2011, pp. 882-890.

[6] M. Zhang, L. Gao, J. Huang, and M. Honig, "Cooperative and competitive operator pricing for mobile crowdsourced internet access," in IEEE INFOCOM, Atlanta, GA, May 2017.

[7] CSIM20, [http://www.mesquite.com].

[8] K. Koufos et al., "Deliverable D5.4: Future spectrum system concept," METIS, vol. Document Number: ICT-317669-METIS/D5.4, April 2015.

[9] P. Caballero, A. Banchs, G. de Veciana, and X. Costa-Perez, "Network slicing games: Enabling customization in multi-tenant networks," in IEEE INFOCOM, Atlanta, GA, May 2017.

[10] V. Sciancalepore, K. Samdanis, and X. Costa-Perez, "Mobile traffic forecasting for maximizing $5 \mathrm{~g}$ network slicing resource utilization," in IEEE INFOCOM, Atlanta, GA, May 2017.

[11] F. Teng, D. Guo, and M. L. Honig, "Sharing of unlicensed spectrum by strategic operators," IEEE J. Sel. Areas Commun., vol. 35, no. 3, pp. 668-679, March 2017.

[12] Z. Guan and T. Melodia, "Cu-lte: Spectrally-efficient and fair coexistence between lte and wi-fi in unlicensed bands," in IEEE INFOCOM, San Francisco, CA, April 2016, pp. 1-9.

[13] 3GPP, "Network sharing; artechecture and functional description," 3GPP TR 23.251, Jun. 2016.

[14] S. C. Liew, C. H. Kai, H. C. Leung, and P. Wong, "Back-of-the-envelope computation of throughput distributions in CSMA wireless networks," IEEE Trans. Mobile Comput., vol. 9, no. 9, pp. 1319-1331, Sep. 2010.

[15] 3GPP, "Physical layer procedures," 3GPP TR 36.213 v13.4, Dec. 2016.

[16] G. Bianchi, I. Tinnirello, and L. Scalia, "Understanding 802.11e contention-based prioritization mechanisms and their coexistence with legacy 802.11 stations," IEEE Network, vol. 19, no. 4, July/Aug. 2005.

[17] M. Luby, "A simple parallel algorithm for the maximal independent set problem," SIAM Journal on Computing, vol. 15, no. 4, 1986.

[18] Technical Report, Dec. 2017. [Online]. Available: https://1drv.ms/b/s!AuONFCNpT0cnenFq163-yNav2m4

[19] G. Chalkiadakis, E. Elkind, E. Markakis, M. Polukarov, and N. R. Jennings, "Cooperative games with overlapping coalitions," Journal of Artificial Intelligence Research, vol. 39, no. 1, pp. 179-216, Sep. 2010.

[20] S. Boyd, N. Parikh, E. Chu, B. Peleato, and J. Eckstein, "Distributed optimization and statistical learning via the alternating direction method of multipliers," Found. Trends Mach. Learn., vol. 3, no. 1, Jan. 2011.

[21] E. Wei and A. Ozdaglar, "On the o $(1=\mathrm{k})$ convergence of asynchronous distributed alternating direction method of multipliers," in IEEE GlobalSIP, Austin, TX. Dec. 2013, pp. 551-554. 